\title{
UAV-Based Collaborative Electronic Reconnaissance Network for 6G
}

\author{
Fucheng Yang $\mathbb{D},{ }^{1}$ Jie Song, ${ }^{1}$ Wei Xiong, ${ }^{1}$ and Xutao Cui $\mathbb{D}^{2}$ \\ ${ }^{1}$ Naval Aeronautical University Aeronautical Operation Service Academy, Yantai 264001, China \\ ${ }^{2}$ Joint Operation Department of Institute of War Study of Academy of Military Science, Beijing 100001, China \\ Correspondence should be addressed to Xutao Cui; 15063882950@139.com
}

Received 6 June 2021; Accepted 10 August 2021; Published 3 September 2021

Academic Editor: Haitao Xu

Copyright (C) 2021 Fucheng Yang et al. This is an open access article distributed under the Creative Commons Attribution License, which permits unrestricted use, distribution, and reproduction in any medium, provided the original work is properly cited.

\begin{abstract}
In unmanned aerial vehicle (UAV) collaborative electronic reconnaissance network, single UAV is always restricted by flyability and sensing capacity; hence, a cooperative network is built to realize the electronic reconnaissance. In this paper, a three-level electronic reconnaissance network is proposed, including the radiation target, UAV-based electronic reconnaissance equipment, and the command center. Each of the UAVs is capable of monitoring several radiation targets at the same time. Since the topology of the UAV network influences the effect of electronic reconnaissance, in this contribution, optimization is achieved based on the improvement of radiation coverage. If there is no radiation target within the sensing scope, the corresponding UAV will remove according to our novel strategy. Iterate operations are carried out for the relative optimum performance. Simulation results show that the UAV network topology optimization is capable of improving the coverage of radiation targets effectively.
\end{abstract}

\section{Induction}

In 2018, the US Federal Communications Commission (FCC) pointed out that $6 \mathrm{G}$ communication era is not far away anymore [1-3]. Coming to 2019, the global 6G summit was held, which defined the general direction and basic features of $6 \mathrm{G}$ development. China officially launched 6G technology research and development at the end of 2019; then, the 6G technology research and development promotion working group was established. In 2020, the standardization of $5 \mathrm{G}$ communication has officially drawn to a close [4]. In order to meet the higher demand in the future, $6 \mathrm{G}$ research and development has been formally discussed on the agenda. Compared to 5G, 6G technology will provide more accurate phase synchronization technology, higher spectrum/energy/cost efficiency, TBPS level transmission efficiency, 1/10 delay, 100x link density, nearly $100 \%$ global coverage, subcentimeter positioning accuracy, and millisecond positioning update rate. As the most important resource of $6 \mathrm{G}$ network, spectrum resource faces new challenges [5]. First of all, the spectrum usage in the $6 \mathrm{G}$ era is characterized by full spectrum, including millimeter wave, terahertz, and visible light. How to realize multispectrum sensing within multisystem is an urgent problem to be solved. Secondly, with the integration of $6 \mathrm{G}$ stereoscopic space, the original planar spectrum resources are divided into the three-dimensional space, and the corresponding spectrum perception ability should also be upgraded from $2 \mathrm{D}$ to $3 \mathrm{D}$. Finally, $6 \mathrm{G}$ communication is affected by environment, channel, platform, load, system, and other factors, and spectrum resources are more fragmented. How to quickly and accurately complete spectrum perception is the most important step to utilize the fragmented spectrum resources.

In view of the abovementioned characteristics of full spectrum, three-dimensional and fragmentation of $6 \mathrm{G}$, we proposed to use UAV platforms to carry out collaborative spectrum sensing of $6 \mathrm{G}$ communication network. As a remote control command or according to its own program control aircraft, unmanned aerial vehicles (UAVs) with spectrum sensing nodes are capable of achieving fast, accurate, and flexible spectrum sensing effect [6-12]. In the past decades, the use and development UAVs has experienced an explosive 
growth as the performance of electronic devices has improved and costs have fallen. Benefiting from the economical cost of UAV, the flexibility of takeoff and landing, and the versatility of its application, UAV and its related technologies have received extensive attention.

As a mobile and flexible flight platform, the UAV has unique advantages by equipped passive electronic reconnaissance equipment and forming a network to carry out collaborative electronic reconnaissance [13-15]. However, the mainly difficult of route planning for collaborative electronic reconnaissance of UAV clusters lies in the high dynamic collaborative planning for targets with unknown radiation sources without any prior knowledge[8, 11, 16-18]. From the perspective of topology planning, generally, the development of sensor network goes through three stages. The first phase is sensor node deployment for a static area, such as mountain fire prevention and environmental monitoring in large petroleum mining areas [5, 19]. This kind of sensor network optimization can be summarized as static coverage method, that is, through reasonable deployment of the sensor network and optimization of its topology structure, the observation target in the detection area can be observed as realistically as possible [20]. Usually, the principle of polygon partitioning is used to partition the sensor network, calculate the best observation position in each partition, and make the sensor as close as possible to this best position, so as to achieve better observation effect. The first method of static optimization of sensor network is suitable for the case that the number of sensor nodes is sufficient and the area of monitoring environment is small, as well as the observation environment is relatively simple [21]. In the face of large area and complex environment, static sensor network needs a huge number of sensor nodes. In this case, it is necessary to plan a reasonable path to realize the patrol of the designated observation area. Compared with the topology optimization of static sensor network, the path planning of the UAV network needs to consider both the strict restrictions of communication link and energy consumption [9]. The route of the UAV cluster is planned based on the static coverage sensor network, and the overall observation environment is divided into several zones. According to the final location of the current zone and the minimum redundancy tendency, the UAV cluster can choose to stay in this zone or go to the next zone. According to the survival/efficiency ratio of UAV considered in literature $[10,15,22,23]$, for environmental perception in extreme situation, route planning is carried out by using the overall survivability and task assignment of the UAV network to guarantee the overall survivability of the UAV network. Another path planning of the UAV network can be summarized as the dynamic UAV network corresponding to the dynamic target group, that is, both the target and the UAV platform have high dynamic capability $[14,24]$. In this case, the path of the UAV platform is not set specifically, but the rules of its movement are designed to adapt to the changing environment. Intelligent dynamic path optimization algorithm has a good development prospect, but at present, intelligent path optimization algorithm is still mainly in the theory, specific to the practical application has not been widely applied.
The limitations of its application are mainly due to the prior knowledge is necessary for the intelligent algorithm to achieve the best optimization. However, the UAV network is often faced with the totally unknown and high dynamic environment. In this case, the intelligent dynamic path planning usually falls into local optimization selection and ignoring the UAV network overall performance. With the change of environment, the optimization based on the partial derivative of often leads to failure.

In the face of dynamic electromagnetic environment, this paper proposes a path optimization method for collaborative electronic reconnaissance using UAV clusters, which can be summarized into two stages. The first stage is load perception, that is, the number of radiation sources within the effective distance perceived by each load. The second stage is platform movement. Explicitly, each platform decides to keep in the original place or move within the effective distance according to the perception effect of the corresponding load and the positions of its neighbors. Finally, the effective coverage rate of the UAV network to the radiation source within the detection range is calculated by simulation under different iteration times, so as to verify the actual effectiveness of the proposed method without prior information.

\section{System Model}

The framework of the cooperative spectrum sensing network in this contribution is a typical triple-layer model widely applied for various scenarios. In this cooperative spectrum sensing network, there are $K$ radiation sources (RSs) monitored by $L$ individual UAV. At the same time, each of the UAVs is capable of sensing several RSs and communicating with the fusion center (FC).

The UAV cluster should cooperate with each other to monitor radiation sources in the designated area. The location setting of the UAV platform should be based on the optimal coverage rate of radiation sources rather than the coverage area, and efforts should be made to match the electronic reconnaissance load carried on the UAVs with the distribution density of radiation sources as far as possible.

As shown in Figure 1, the optimization process of UAV cluster cooperative electronic reconnaissance topology can be divided into two steps: the first step is self-perception of UAV platform load. The second step is to adjust the position of each UAV according to its own perception and the congestion degree feedback by the system. In the process of topology optimization of the UAV cluster, the electronic reconnaissance payload carried by the UAV conducts reconnaissance on the radiation source. The reconnaissance range is a sphere with radius $R$ centering on itself, the single movement distance of the UAV is $D$, and the number of optimization iterations is $N$. The coverage of radiation sources is used as a parameter to evaluate the topology of the UAV network, that is, the ratio of the radiation sources covered by the UAVs in the observation area to the total number of all radiation sources. Due to the complexity of flight environment, factors such as air flow are likely to impact on the UAV network topology. Due to the radiation signal 


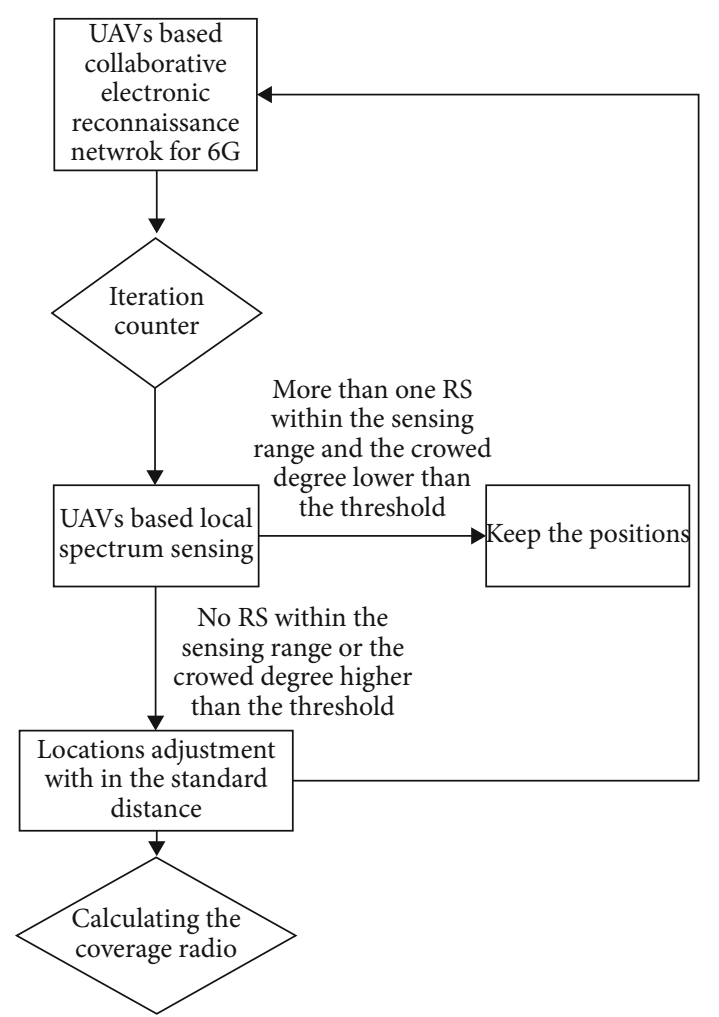

Figure 1: Processing of UAV network topology optimization algorithm.

transmitted through the effects of fading and noise, the coverage of electronic reconnaissance is reduced. In this case, we try to improve the coverage of electronic reconnaissance through the UAV network topology reconstruction. To avoid resource waste, we introduce two concepts: superposition number of radiation source coverage and crowding degree of UAVs, which are the number of each radiation source monitored by UAVs and the number of UAVs near a certain location. Specifically, the superposition number of radiation source coverage can be expressed as follows: $N_{k}$ $=\sum_{l=1}^{L} J\left\{d\left(s_{k}, u_{l}\right) \leq R\right\}$, where $J\{\bullet\}$ is a step function; if the distance between the $k$ th radiant source and the $l$ th UAV platform is less than the perceived distance, it is denoted as 1 ; otherwise, it is denoted as 0 . The congestion degree near the $l$ th UAV within the observation range can be measured by the congestion degree, i.e., $\rho_{l}=N_{l} / L$, where $N_{l}$ is the number of UAV within the perception range of the $l$ th UAV.

\section{Topology Optimization Algorithm}

The path optimization algorithm process of UAV cluster collaborative electronic reconnaissance processes as follows: The number of radiation sources within the perceived range of the $l$ th UAV is first calculated as $N_{\text {sensing }}(l)$, and if $N_{\text {sensing }}(l)=0$, the first step is performed; otherwise, if $N_{\text {sensing }}(l)>0$, the second step is performed.

For the case of $N_{\text {sensing }}(l)=0$, the specific operation can be subdivided into the following three cases:
(1) $N_{(l)}=0$, which means the number of UAVs within the perceived range of the first UAV is 0

In this case, the UAV randomly chooses the distance to move in any direction not exceeding the standard step $D_{\text {step }}$, and the position after moving $\overrightarrow{D^{\prime}}$ can be expressed as follows:

$$
\frac{\overrightarrow{D^{\prime}}=\overrightarrow{D+} \operatorname{rand}\left(D_{\text {step }}\right) \overrightarrow{D^{\prime}}-\vec{D}}{\left|\overrightarrow{D^{\prime}}-\vec{D}\right|,}
$$

where rand $\left(D_{\text {step }}\right)$ denotes the random distance between zero and the standard step $D_{\text {step }}$. If the number of perceived emitters $N_{\text {sensing }}(l)$ increases after moving to a new position $\overrightarrow{D^{\prime}}$, the corresponding UAV will stop moving; otherwise, it will continue to move in accordance with the above rules.

(2) $N_{(l)}=1$, which means there is one UAV platform within the sensing range of the $l$ th UAV. For the convenience of description, we assume that the serial number of the UAV is $i$. At this point, if $N_{\text {sensing }}(i)$ $>N_{\text {sensing }}(l)$ as well as $\rho_{i}=N_{i} / L<\lambda$, which indicates that the perceived number of radiation sources on the $i$ th UAV platform is greater than that of the $l$ th UAV platform, and the crowding degree at the $i$ th UAV platform is lower than the threshold value. In this case, the $l$ th UAV platform moves one step towards the UAV platform:

$$
\frac{\overrightarrow{D^{\prime}}=\overrightarrow{D+} \operatorname{rand}\left(D_{\text {step }}\right) \overrightarrow{D_{i}}-\vec{D}}{\left|\overrightarrow{D_{i}}-\vec{D}\right|}
$$

(3) $N_{(l)}>1$ which means there is more than one UAV platforms within the sensing range of the lth UAV. Firstly, we find the central location of the nearby UAV cluster $\overrightarrow{D_{c}{ }^{\prime}}=\sum_{m \in M} \overrightarrow{D_{m}{ }^{\prime}} / M$. Then, the perceived number of radiation sources at this central location and the platform congestion are calculated, respectively:

$$
N_{\text {sensing }}(c)=\frac{\sum_{m \in M} N_{\text {sensing }}(m)}{M} .
$$

Then, the coverage radio is given by

$$
\rho_{c}=\frac{\sum_{m \in M} \rho_{m}}{M}
$$




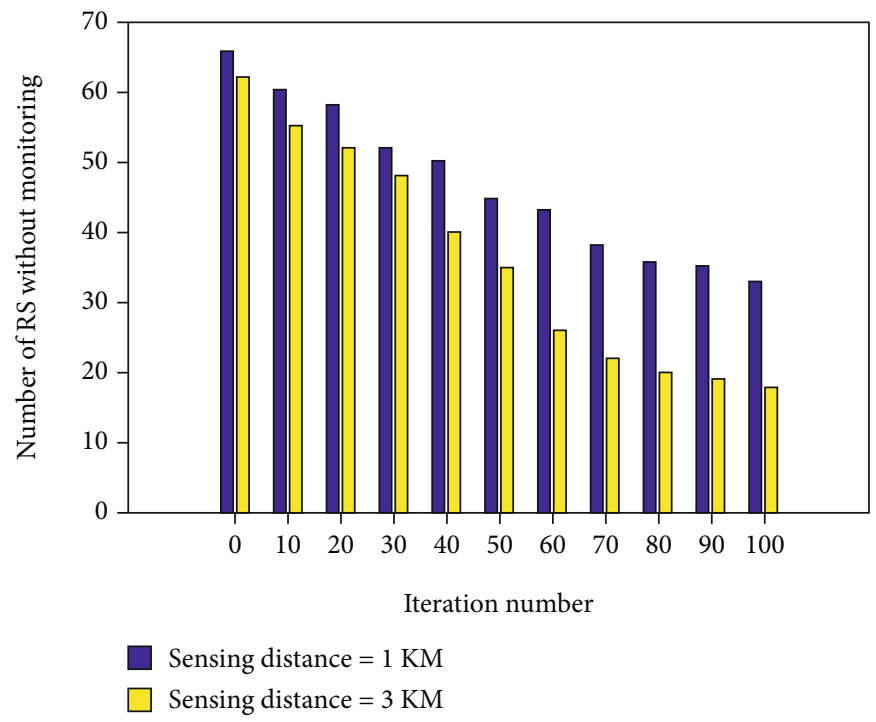

FIGURE 2: Number of RSs without monitoring versus iteration operations number for 30 UAVs with the effective distance of $1 \mathrm{~km}$ or $3 \mathrm{~km}$.

\section{Performance Analysis}

In this section, we focus on the analysis of the coverage of radiation sources, which is mentioned at the beginning of this article. Explicitly, each of the UAVs indicates whether there is a RS within its sensing range and then transmutes its local decision to the fusion center (FC). The coverage of RS is used as a parameter to evaluate the topology of the UAV network, that is, the ratio of the radiation sources covered by the UAVs in the observation area to the total number of all RSs. In the transmission process, it is assumed that in the consecutive time slots, the UAV senses the RSs in the first time slot and then forwards the local results to the FC in the second time slot. It is assumed that the location of the UAV is constant and does not change in each time slot. For convenience of description, the coordinate of the FC and the $i$ th UAV is set as $(0,0,0)$ and $\left(x_{i}^{U}(t), y_{i}^{U}(t), z_{i}^{U}\right.$ $(t))$, respectively. Hence, the corresponding distance can be denoted as follows:

$$
d_{i}^{U F}(t)=\sqrt{\left(x_{j}^{U}(t)\right)^{2}+\left(y_{j}^{U}(t)\right)^{2}+\left(z_{j}^{U}(t)\right)^{2}}
$$

At one time slot, the coordinate of the $j$ th RS and the $i$ th UAV is expressed as $\left(x_{j}^{R}(t), y_{j}^{R}(t), z_{j}^{R}(t)\right)$ and $\left(x_{i}^{U}(t)\right.$, $\left.y_{i}^{U}(t), z_{i}^{U}(t)\right)$, respectively. The distance between the $j$ th RS and the $i$ th UAV is $d_{i j}^{U R}(t)=$ $\sqrt{\left(x_{i}^{U}(t)-x_{j}^{R}(t)\right)^{2}+\left(y_{i}^{U}(t)-y_{j}^{R}(t)\right)^{2}+\left(z_{i}^{U}(t)-z_{j}^{R}(t)\right)^{2}}$. Compared with the nonline-of-sight (NLOS), line-of-sight (LOS) plays much more important part in the communication and sensing of UAVs. It is reasonable to assume that at the $t$ th time slot, the $j$ th RS transmission power is sensed by the ith UAV, and then, the UAV forwards its local indication to the FC.
Explicitly, the path loss of the LOS between the $j$ th RS and the $i$ th UAV can be expressed as

$$
\mathrm{PL}_{i j}^{U R}(t)=L_{i j}^{U R}(t)+20 \log \left(d_{i j}^{U R}(t)\right)+\eta
$$

where $L_{i j}^{U R}(t)=20 \log (f)+20 \log (4 \pi / c)$ is the free pass lose based on the radio frequency of $f$ and $\eta$ represents the additional loss of LOS. It is reasonable to assume that the threshold of all the UAVs is the same and sets as $\mathrm{Th}_{U}$. Then, the possibility of the $i$ th UAV discovers the $j$ th RS can be represented as $P_{i j}^{U R}=P\left(\mathrm{PL}_{i j}^{U R}>\mathrm{Th}_{U}\right)$.

Similarly, the path loss of the LOS between the ith UAV and the FC can be denoted as

$$
\mathrm{PL}_{i}^{U F}(t)=L_{i}^{U F}(t)+20 \log \left(d_{i}^{U F}(t)\right)+\eta
$$

The possibility of the FC is capable of estimating the UAV's sensing result can be written as $P_{i}^{U F}=P\left(\mathrm{PL}_{i}^{U F}\right.$ > $\mathrm{Th}_{F}$ ), where $\mathrm{Th}_{F}$ is the SNR threshold of the FC.

For the $j$ th RS, the probability th is covered by the UAV network when both

The probability that the $j$ th RS is observed by the FC through the sensing of the $i$ th UAV equals to $P_{i j}^{U R} P_{i}^{U F}$.

Consequently, the overall probability that the $j$ th RS is out of coverage of the UAV network can be calculated as

$$
P_{j}^{\text {out }}=\prod_{i=1}^{\mathrm{I}}\left(1-P_{i j}^{U R} P_{i}^{U F}\right) .
$$

From Equation (8), we can see that when the radio frequency and communication environment is set, the ratio of coverage is only affected by the location of each UAV. 


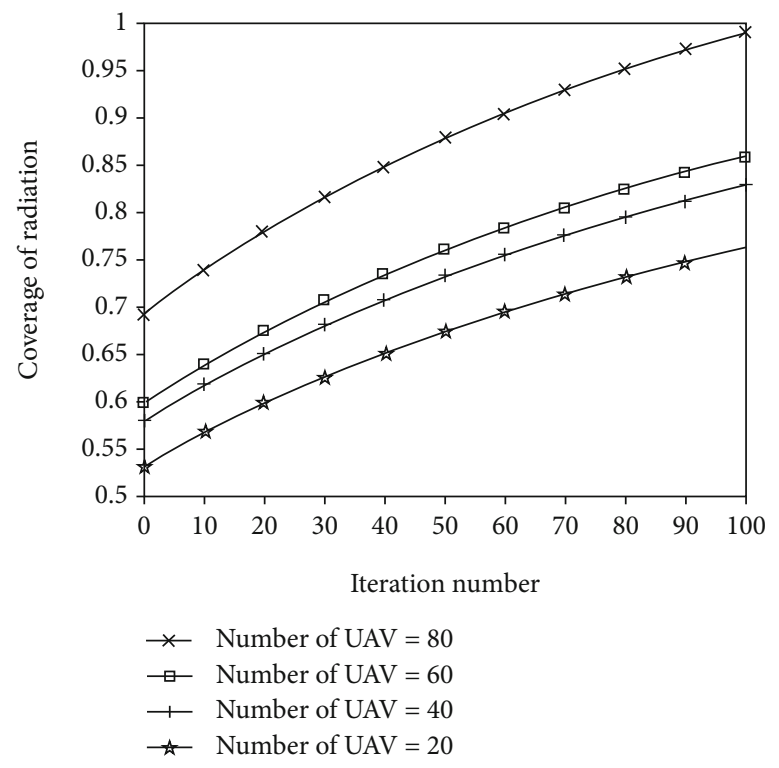

FIgURE 3: Coverage ratio of radiation versus iteration number for various number of UAVs with $0.5 \mathrm{~km}$ sensing distance.

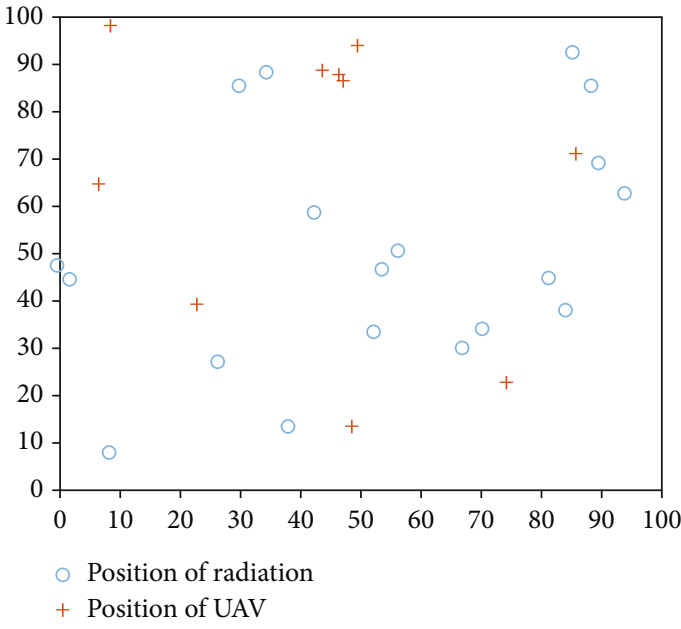

(a)

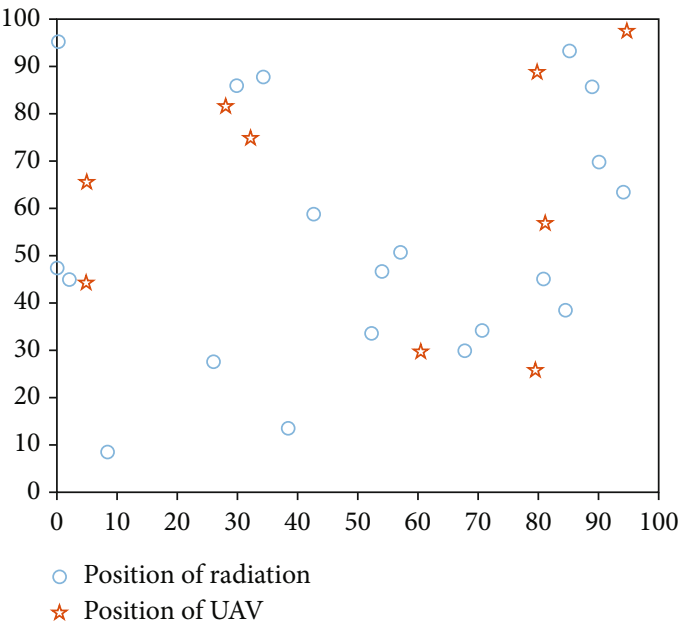

(b)

FIgURE 4: (a) Topology of the UAV network without iteration operations. (b) Topology of the UAV network after 20 iteration operations.

\section{Simulation Results}

In this section, for the optimization method of UAV cluster topology structure, the Monte Carlo method is adopted to realize the optimization and verification of radiation source coverage. Several iterative operation experiments are carried out, and the reliability of the optimization algorithm is discussed and analyzed by using MATLAB. In the simulation process, we assume that the load carried on the UAV platform is sensitive and effective and has the same uniform performance. In the initial step, the UAVs are randomly distributed in the designated area, and the subsequent position is adjusted according to the topology optimization algorithm.

In Figure 2, we show the utility of sensing distance and the number of iterations. In our simulation, $100 \mathrm{RSs}$ are distributed in $50 \mathrm{~km} \times 50 \mathrm{~km} \times 10 \mathrm{~km}$ space randomly. There are 30 UAVs used to sense the RS's states with the effective distance of $1 \mathrm{~km}$ or $3 \mathrm{~km}$. It can be seen that the number of RS without monitoring decreases as the number of iteration optimization grows. When the iterative optimization number equals to 0 , the scenario means that the locations of UAVs and the RSs are randomly generated. Figure 2 shows that our proposed topology optimization approach is effective to decrease the number of RSs without monitored. Both the sensing distance and the iteration operations affect the performance of UAV networks significantly. However, the performance of the UAV network tends to be stable when the number of iteration operations reaches 70 .

As shown in Figure 3, we assume that $50 \mathrm{RSs}$ are randomly distributed in a cube with length of $20 \mathrm{~km}$, and the UAV cluster carries out electronic reconnaissance in this space. The effective perceived distance of the UAV carrying the electronic surveillance payload is $0.5 \mathrm{~km}$. Each UAV 
platform sends the results of its electronic surveillance to the FC, which adjusts its position according to the effectiveness of the electronic surveillance to achieve better RS coverage radio. As shown in Figure 3, increasing the number of UAV platforms can significantly improve the coverage ratio of UAV cluster network collaborative electronic reconnaissance, but it will bring about an increase in system cost and system complexity. According to the UAV swarm adaptive topology optimization method proposed by us, the UAV can adjust its position in real time according to its own RS perception and the position of the surrounding UAV platforms. Through the iterative adjustment method, the effective coverage ratio of RSs within the perceived range can be improved slightly without increasing the number of platforms and the complexity of the system.

By comparing Figures 4(a) and 4(b), we can see that the UAV platform can adjust its position by itself without the position information of the radiation source, thus optimizing the topological structure of the UAV cluster. The simulation results show that after 20 iteration movements of topology optimization, UAVs' position distribution is more reasonable, and the corresponding RS coverage ratio is also improved.

\section{Conclusion}

In this paper, we establish a UAV network to realize collaborative electronic reconnaissance for $6 \mathrm{G}$. In the absence of a priori information, we proposed a method to optimize the autonomous topology of the cluster based on the number of surrounding emitters and the number of nearby UAVs sensed by the UAV platform. By setting the perceptual threshold and the number of iterations of network topology optimization, the overall control of the cluster is realized. Through the simulation results, we can see that under reasonable parameter settings, the proposed optimization method of UAV cluster collaborative electromagnetic sensing topology structure can effectively improve the coverage ratio of the UAV cluster to the overall RS. At the same time, the cluster performance and system complexity are effectively balanced by setting the threshold and iteration times.

\section{Data Availability}

The simulation data used to support the findings of this study are available from the corresponding author upon request.

\section{Conflicts of Interest}

The authors declare that there is no conflict of interest regarding the publication of this article.

\section{Acknowledgments}

This work has been supported by the National Natural Science Foundation of China (grant numbers: NSFC51790554, NSFC-61971433, and NSFC-62001499). This work was also supported in part by Sichuan Science and Technology Program under Grant 2020YFH0011.

\section{References}

[1] M. B. H. Weiss, K. Werbach, D. C. Sicker, and C. E. C. Bastidas, "On the application of blockchains to spectrum management," IEEE Transactions on Cognitive Communications and Networking, vol. 5, no. 2, pp. 193-205, 2019.

[2] K. Daniel and C. Wietfeld, "Using public network infrastructures for UAV remote sensing in civilian security operations," DORTMUND UNIV (GERMANY FR), 2011.

[3] L. Li, Q. Cheng, K. Xue, C. Yang, Z. Han, and Z. Han, "Downlink transmit power control in ultra-dense UAV network based on mean field game and deep reinforcement learning," IEEE Transactions on Vehicular Technology, vol. 69, no. 12, pp. 15594-15605, 2020.

[4] F. Santoso, M. A. Garratt, and S. G. Anavatti, "State-of-the-art intelligent flight control systems in unmanned aerial vehicles," IEEE Transactions on Automation Science and Engineering, vol. 15, no. 2, pp. 613-627, 2018.

[5] G. Chmaj and H. Selvaraj, "Distributed Processing Applications for UAV/Drones: A Survey," in Progress in Systems Engineering, pp. 449-454, Springer, Cham, 2015.

[6] A. D. Dang, H. M. La, and J. Horn, "Distributed formation control for autonomous robots following desired shapes in noisy environment," in Multisensor Fusion and Integration for Intelligent Systems (MFI), International Conference on IEEE, pp. 285-290, 2016.

[7] H. Hmam, "Optimal sensor velocity configuration for TDOAFDOA Geolocation," IEEE Transactions on Signal Processing, vol. 65, no. 3, pp. 628-637, 2017.

[8] G. Pandurangan, P. Robinson, and A. Trehan, "Dex: selfhealing expanders," Distributed Computing, vol. 29, no. 3, pp. 163-185, 2016.

[9] L. Li, M. Wang, K. Xue et al., "Delay optimization in multiUAV edge caching networks: a robust mean field game," IEEE Transactions on Vehicular Technology, vol. 70, no. 1, pp. 808819, 2021.

[10] J. Lee, K. Kim, S. Yoo et al., "Constructing a reliable and fast recoverable network for drones," in IEEE International Conference on Communications (ICC), vol. 20, pp. 1-6, 2016.

[11] R. Ladig, H. Paul, R. Miyazaki, and K. Shimonomura, "Aerial manipulation using multirotor UAV: a review from the aspect of operating space and force," Journal of Robotics \& Mechatronics, vol. 33, no. 2, pp. 196-204, 2021.

[12] L. Li, H. Ren, Q. Cheng et al., "Millimeter-wave networking in sky: a machine learning and mean field game approach for joint beamforming and beam-steering," IEEE Transactions on Wireless Communications, vol. 19, no. 10, pp. 6393-6408, 2020.

[13] N. Zhang and N. Cheng, "Gamage A T, Zhang K, Mark J W, Shen X, Cloud assisted HetNets toward $5 \mathrm{G}$ wireless networks, IEEE Communication," Magazine, vol. 53, no. 6, pp. 59-65, 2015.

[14] N. Zhao, W. Lu, M. Sheng et al., "UAV-assisted emergency networks in disasters," IEEE Wireless Communications, vol. 26, no. 1, pp. 45-51, 2019.

[15] F. Tang, Y. Zhou, and N. Kato, "Deep reinforcement learning for dynamic uplink/downlink resource allocation in high 
mobility 5G HetNet," IEEE Journal on Selected Areas in Communications, vol. 38, no. 12, pp. 2773-2782, 2020.

[16] Q. Wu and R. Zhang, "Common throughput maximization in UAV enabled OFDMA systems with delay consideration," IEEE Transactions on Communications, vol. 66, no. 12, pp. 6614-6627, 2018.

[17] X. Liu, Y. Liu, Y. Chen, and L. Hanzo, "Trajectory design and power control for multi-UAV assisted wireless networks: a machine learning approach," IEEE Transactions on Vehicular Technology, vol. 68, no. 8, pp. 7957-7969, 2019.

[18] Q. Zhang, M. Jiang, Z. Feng, W. Li, W. Zhang, and M. Pan, "IoT enabled UAV: network architecture and routing algorithm," IEEE Internet of Things Journal, vol. 6, no. 2, pp. 3727-3742, 2019.

[19] M. Mozaffari, W. Saad, M. Bennis, Y. H. Nam, and M. Debbah, "A tutorial on UAVs for wireless networks: applications, challenges, and open problems," in IEEE communications surveys \& tutorials, vol. 21, pp. 2334-2360, 2019.

[20] S. Haykin, "Artificial intelligence communicates with cognitive dynamic system for cybersecurity," IEEE Transactions on Cognitive Communications and Networking, vol. 5, no. 3, pp. 463-475, 2019.

[21] Y. Xu and C. Wang, "Load-aware dynamic spectrum access for small-cell networks: a graphical game approach," IEEE Transactions on Vehicular Technology, vol. 65, no. 10, pp. 87948800, 2015.

[22] Y. Gu, Y. Huang, H. Hu, W. Gao, and Y. Pan, "Energy efficiency optimization of cognitive UAV-assisted edge communication for semantic Internet of Things," Wireless Communications and Mobile Computing, vol. 1, no. 1, 12 pages, 2021.

[23] M. Razzaque and S. Dobson, "Energy-efficient sensing in wireless sensor networks using com-pressed sensing," Sensors, vol. 14, no. 2, pp. 2822-2859, 2014.

[24] L. Li, J. Huang, Q. Cheng, H. Meng, and Z. Han, “Automatic modulation recognition: a few-shot learning method based on the capsule network," IEEE Wireless Communications Letters, vol. 10, no. 3, pp. 474-477, 2021. 